\title{
Th17 Cells Pathways in Multiple Sclerosis and Neuromyelitis Optica Spectrum Disorders: Pathophysiological and Therapeutic Implications
}

\author{
Giordani Rodrigues Dos Passos, ${ }^{1}$ Douglas Kazutoshi Sato, ${ }^{1,2,3}$ \\ Jefferson Becker, ${ }^{1}$ and Kazuo Fujihara ${ }^{3}$ \\ ${ }^{1}$ Brain Institute and Department of Neurology, Faculty of Medicine, Pontifical Catholic University of Rio Grande do Sul (PUCRS), \\ Avenida Ipiranga 6690, 90610-000 Porto Alegre, RS, Brazil \\ ${ }^{2}$ Department of Neurology and LIM-15, Faculty of Medicine, University of Sao Paulo (USP), Avenida Dr. Arnaldo 455, \\ 01246-903 São Paulo, SP, Brazil \\ ${ }^{3}$ Department of Multiple Sclerosis Therapeutics, Tohoku University Graduate School of Medicine, 1-1 Seiryo-machi, Aobaku, \\ Sendai, Miyagi 980-8574, Japan \\ Correspondence should be addressed to Douglas Kazutoshi Sato; douglas.sato@med.tohoku.ac.jp
}

Received 25 September 2015; Revised 22 December 2015; Accepted 24 December 2015

Academic Editor: Musheng Bao

Copyright (C) 2016 Giordani Rodrigues Dos Passos et al. This is an open access article distributed under the Creative Commons Attribution License, which permits unrestricted use, distribution, and reproduction in any medium, provided the original work is properly cited.

\begin{abstract}
Several animal and human studies have implicated CD4+ T helper 17 (Th17) cells and their downstream pathways in the pathogenesis of central nervous system (CNS) autoimmunity in multiple sclerosis (MS) and neuromyelitis optica spectrum disorders (NMOSD), challenging the traditional Th1-Th2 paradigm. Th17 cells can efficiently cross the blood-brain barrier using alternate ways from Thl cells, promote its disruption, and induce the activation of other inflammatory cells in the CNS. A number of environmental factors modulate the activity of Th17 pathways, so changes in the diet, exposure to infections, and other environmental factors can potentially change the risk of development of autoimmunity. Currently, new drugs targeting specific points of the Th17 pathways are already being tested in clinical trials and provide basis for the development of biomarkers to monitor disease activity. Herein, we review the key findings supporting the relevance of the Th17 pathways in the pathogenesis of MS and NMOSD, as well as their potential role as therapeutic targets in the treatment of immune-mediated CNS disorders.
\end{abstract}

\section{Introduction}

Multiple sclerosis (MS) is a chronic immune-mediated demyelinating disease of the central nervous system (CNS) characterized by a relapsing-remitting (RR) or a progressive course with multifocal CNS dysfunctions [1]. Neuromyelitis optica spectrum disorders (NMOSD) include the entity previously known as neuromyelitis optica (NMO) and patients with limited forms (e.g., only myelitis or optic neuritis) and comprise a phenotypic continuum of primarily immunemediated astrocyte injury, rather than a primary demyelinating disease, with preferential involvement of the optic nerves, brainstem, and the spinal cord $[2,3]$.
The nosology of NMO remained controversial for more than one century after its first description, by Devic, in 1894 [3]. It was speculated that it could represent a topographically restricted severe MS variant. A considerable advance in the understanding of those disorders was the identification of pathogenic autoantibodies against aquaporin-4 (anti-AQP4$\mathrm{IgG}$ ) in patients with NMO, which allowed for the establishment of NMO as a distinct nosological entity [3]. Despite the fact that both diseases have an inflammatory process restricted to the CNS and a relapsing course in the majority of patients, there are major differences in clinical definition and understanding of the two diseases. Astrocyte injury leading to secondary demyelination is the hallmark of NMO, at 
least in those patients who are AQP4-IgG-seropositive, while primary demyelinating lesions with $\mathrm{T}$ cell and macrophage infiltration are seen in MS [2]. From the clinical and radiological standpoint, both disorders may present optic neuritis, transverse myelitis, and/or demyelinating brain lesions, but some features are specially suggestive of NMO, such as bilateral optic neuritis, involvement of the optic chiasm, or severe residual visual loss; a complete transverse myelitis, usually with longitudinally extensive lesions on the MRI; and an area postrema syndrome, characterized by intractable nausea, vomiting, and hiccups [3]. Besides that, it has been shown that several immunological therapies commonly used for MS fail to control or even increase disease activity in NMOSD [4], thus suggesting a distinct underlying pathophysiological process in each of those disorders and highlighting the need for a precise distinction between them in order to avoid the potentially harmful consequences of a misdiagnosis.

In both MS and NMOSD, T-B cell interaction has been pointed out as an important factor in the genesis of the disease process. In especially MS, increasing therapeutic options became available in recent years, and some of them involve control of autoreactive $\mathrm{T}$ cells, which highlights the importance of further understanding of the role of each of those cell types. Some knowledge about immune mechanisms involving autoreactive $\mathrm{T}$ cells comes from experimental autoimmune encephalomyelitis (EAE), the animal model of MS, and from animal models using passive human antiAQP4-IgG transfer in NMO.

Initially, the group of $\mathrm{CD} 4+\mathrm{T}$ lymphocytes known as helper $\mathrm{T}(\mathrm{Th})$ cells was believed to differentiate into two mutually exclusive phenotypes: type 1 ones (Thl), which are classically induced by interleukin- (IL-) 12 and produce interferon gamma (IFN- $\gamma$ ), and type 2 ones (Th2), which are stimulated and secrete IL-4 [5]. At that time, the Thl pathway, regarded as proinflammatory, was considered to be the most important mediator of the pathogenesis of both EAE and MS, while the Th2 pathway would have an antagonist effect on Th1 cells and, consequently, a beneficial effect on the disease process [6]. However, subsequent studies provided consistent evidence that mice were still susceptible to EAE after genetic ablation of key cytokines of the Thl pathway, such as IFN$\gamma$, indicating that other unknown pathways were involved [7].

More recently, a new phenotype of Th cells was described, namely, Th17, whose signature cytokine is the IL-17 family. Th17 cells have been implicated in several autoimmune disorders and these cells seem to be relevant in the development of CNS autoimmunity. Here, we review the key findings from animal and human studies supporting the role of Th17 pathways in the MS and NMOSD pathogenesis and potential therapeutic targets under clinical investigation.

\section{Brief Review of the Th17 Pathways}

In 2005, it was demonstrated that näive Th cells would differentiate into a new lineage called Th17, due to its capacity to produce large amounts of IL-17 $[8,9]$. Currently, the cytokine previously named as IL-17 is referred to as IL-17A, since it became clear that IL-17 actually represents a family of cytokines, which includes IL-17A to IL-17F.

The process of differentiation of näive Th cells into Th17 is dependent on IL-23 [10] and potently inhibited by IFN $-\gamma$ and IL-4 [8]. IL-23 knockout mice are resistant to EAE and lacked Th17 cells [11], suggesting that the Th17 pathway is implicated in the pathogenesis of EAE. However, the differentiation of T näive cells into Th17 cells may be induced not only by IL-23, but also by the combination of transforming growth factor beta 1 (TGF- $\beta 1$ ) and IL-6. Moreover, IL- $1 \beta$, in combination with IL- 6 and IL-23 and independently of TGF- $\beta 1$, may induce a different (and pathogenic) phenotype of Th17 cells, characterized by the coexpression of RAR-related orphan receptor gamma t $(\mathrm{ROR} \gamma \mathrm{t})$ and T-bet [12].

Indeed, Th17 cells can present different phenotypes, pathogenic or not, according to the modulating factors they are exposed to, such as IL-23, and the differential expression of some cytokines and chemokines, such as IL$17 \mathrm{~A}, \mathrm{IL}-17 \mathrm{~F}, \mathrm{IL}-21$, and ROR $\gamma \mathrm{t}$ transcription factor [13]. Moreover, Th17 cells have impressive plasticity, that is, ability to transition between different phenotypes throughout their life span [7]. It has been demonstrated that a significant proportion of IL-17-producing (Th17) cells converts into IFN- $\gamma$-producing $\mathrm{T}$ cells, partially due to IL-23-mediated reprogramming [14]. There is also a subset of Thl7 cells that, in addition to IL-17A, simultaneously express IFN- $\gamma$ and have chemokine receptors from both Th17 and Thl cells [15].

Granulocyte macrophage colony stimulating factor (GMCSF) is a growth factor that acts as a proinflammatory cytokine and is critically involved in Th17 and other cellmediated immune responses. It is produced by several different cells, especially T cells, in response to IL-23 and IL-1 $\beta[16]$ and induces the activation, maturation, and differentiation of macrophages and of dendritic cells (which secrete IL-23 and IL-6) [17]. Notably, there is a positive feedback loop between GM-CSF and IL-23, which plays a critical role in the expansion of pathogenic Th17 cells. Indeed, studies with EAE have shown that GM-CSF is essential for mediating Th17 cells-induced encephalitogenicity $[16,18,19]$. Recently, another study with EAE has suggested that the GM-CSF-producing $\mathrm{T}$ cells likely represent a distinct subset of $\mathrm{T}$ helper cells, designated as Th-GM [20].

Currently, after several studies indicating that the IL-17 family plays a crucial role in the development of EAE [21, 22], the pathogenic potential of Th17 pathways, in addition to that of Thl pathways, in the development of EAE has been widely accepted, although not fully understood. Th1-induced EAE presents the classical phenotype, characterized by an ascending caudocranial paralysis, while Th17 polarization induces EAE with an atypical phenotype, characterized by ataxic gait [23].

Based on the previously mentioned findings, the classical Th1 paradigm was replaced by the Th1/Th17 paradigm in both EAE and MS. It is postulated that Thl7 cells would play a more relevant role in the initial phases of EAE and MS, while Th1 cells would be more important in later stages of the inflammation in the CNS [6]. 


\section{Modulation of Th17 Responses by Environmental Factors}

Relevant modulation of Th17 responses occurs in mucosal tissues, especially those of the lungs [24] and gastrointestinal tract [25]. This modulation is dependent on the complex interaction of local immune elements with a multitude of pathogens, nutritional components, and other environmental factors. It is believed that $\mathrm{T}$ cell clones stimulated by these interactions in the periphery would induce activated $\mathrm{T}$ cells able to migrate through the blood-brain barrier (BBB) and induce damage in the CNS. Further studies are still required to better understand the role of environmental factors in MS and other autoimmune diseases, but this growing amount of evidence can provide new targets for therapeutic interventions.

One of the mechanisms of interaction between environmental factors and $\mathrm{T}$ cells is the aryl hydrocarbon receptor (AHR), which modulates the differentiation towards either Th17 or regulatory $\mathrm{T}$ (Treg) cells, increasing or decreasing, respectively, the severity of EAE, depending on its ligand [26]. Different compounds from the environment, including commensal microbiota and human pathogens, can act as a ligand for the AHR; moreover, such compounds may act only locally (i.e., in the mucosal tissues) or reach the circulation, causing changes in the immune system of different compartments [7].

Traditionally, systemic infections (especially by viral agents) have been believed to play a role in triggering or modulating the immune process that ultimately may lead to autoimmune CNS diseases. More recently, however, several studies have pointed out that, under special circumstances, normal intestinal microbiota may also activate previously quiescent autoreactive T cells in the gut-associated lymphoid tissues (GALT), thus precipitating autoimmunity [25]. There is some evidence implicating the small intestine as the major site for activation of effector Th1 and Th17 cells and segmented filamentous bacteria as the major inducers of IL-17-producing immune cells $[27,28]$. As an example, cell cultures derived from NMO patients showed a higher Th17 responsiveness to Escherichia coli, associated with elevated IL-1 $\beta$, IL-6, and IL17 production and decreased IL-10 release, when compared to healthy controls [29]. Besides the complex regulation by cytokines and commensal or pathogenic microorganisms in $\mathrm{T}$ cell differentiation and function, Th cells are also regulated (and can be dynamically reprogrammed) by cellular metabolic pathways, including those related to glucose, amino acid, and lipid metabolism [30, 31]. Further studies are required to investigate the role of gut microbiota in the pathogenesis of MS and NMO.

Moreover, high concentrations of sodium chloride and high dietary salt intake have also been shown to enhance the differentiation of Th17 cells and lead to a more severe form of EAE [32]. That phenomenon seems to be mediated by serum glucocorticoid kinase 1 (SGK1), a downstream molecule of IL-23 signaling. SGK1 expression increases after elevation of salt concentration, promoting IL-23R expression and subsequently enhanced Th17 cell differentiation and autoimmunity development [33]. Interestingly, a recently published prospective study has found a positive correlation between higher sodium intake and increased disease activity, measured by both clinical and radiological parameters in patients with MS [34]. Further studies are required to evaluate if the large amounts of salt intake could increase the risk of developing MS.

Finally, substance P, a stress-related neuropeptide, was shown to influence T cell and cytokine profiles in cell cultures derived from patients with generalized anxiety disorder [35]. Complex T cell functional dysregulation (including Th1 and Th2 deficiency and Th17 hyperactivation) was further enhanced by substance $P$, thus suggesting rationale for the influence of chronic stress and anxiety disorders in individuals' susceptibility to autoimmune disease [35]. However, such influence of substance $\mathrm{P}$ in immune function has not been studied in MS or NMOSD yet.

\section{Th17 as Pioneering Cells in the Breakdown of the BBB}

After modulation and selection of T cells in peripheral tissues, pathogenic autoreactive T cells need to cross the BBB in order to cause inflammation into the CNS. Th17 cells have a large number of chemokines and chemokine receptors required to cross the $\mathrm{BBB}$, which enables them to disrupt the $\mathrm{BBB}$ and access the CNS via several different pathways. In vitro and in vivo studies have shown that, through the action of IL-17A and IL-22, Th17 cells can efficiently disrupt BBB tight junctions, express high levels of the cytolytic enzyme granzyme $\mathrm{B}$, and promote the recruitment of additional CD4+ lymphocytes from the systemic circulation into the CNS [36]. Th17 cells are also able to induce CXCL1 and CXCL2, chemokines that are potent attractants for polymorphonuclear cells and play an important role in the breakdown of the BBB in EAE [37]. Th17 cells can access the subarachnoid space via upregulation of the CCR6 receptor, expressed in the epithelium of the choroid plexus, in a process that is critical for the initiation of EAE [38]. Moreover, IL-17A is a key factor in the breakdown of the BBB by direct impairment of its integrity due to the formation of reactive oxygen species within the endothelial cells [39]. Evidences from EAE demonstrate that Th1 cells preferentially access the CNS by using the $\alpha 4 \beta 1$ integrin, while Th17 cells do that by means of the $\alpha \mathrm{L} \beta 2$ integrin (LFA-1) [40]. Melanoma cell adhesion molecule (MCAM) or CD146 is another adhesive molecule expressed by Th17 cells, but not by Th1 cells [41].

Some T cells can secrete both IL-17 and IFN- $\gamma$, being called Th1/Th17 cells. These cells can infiltrate the CNS early in the course of EAE and may be involved in microglial activation and thus may have an important role in the development of the disease [42]. In addition, increased proportions of Th1/Th17 cells were found in the blood and in the brain tissue of MS patients [15]. This amount of evidence suggests that Th17 cells act as pioneering cells in the induction phase of EAE and, presumably, in the early phases of MS.

\section{Th17 in MS}

Following studies that suggested the role of Th17 responses in EAE, several findings from different groups using different 
TABLE 1: Hallmarks on the understanding of the role of the Th17 pathways in MS.

\begin{tabular}{lc}
\hline Finding & Reference \\
\hline $\begin{array}{l}\text { Increased IL-17 found in the blood and CSF of RRMS } \\
\text { patients, especially during relapse }\end{array}$ & {$[43]$} \\
\hline \begin{tabular}{l} 
IL-17-producing T cells identified in EAE \\
\hline $\begin{array}{l}\text { Increased Th17 cells and IL-17 found in the brain of MS } \\
\text { patients }\end{array}$
\end{tabular} & {$[48]$} \\
\hline $\begin{array}{l}\text { IL-17 production correlates with MRI activity } \\
\text { Secukinumab (anti-IL-17A monoclonal antibody) } \\
\text { reduces MRI lesions in a phase II clinical trial }\end{array}$ & {$[114]$} \\
\hline
\end{tabular}

techniques have provided substantial evidence that the Th17 pathways play a critical role in MS (a summary of the most important studies provided in Table 1).

An increased frequency of Th17 cells is detected in the peripheral blood and cerebrospinal fluid (CSF) of some RRMS and clinically isolated syndrome (CIS) patients, especially during the acute episode, when compared to patients with noninflammatory neurological diseases [4347]. Increased proportion of Th17 cells, as well as increased levels of IL-17A (protein and messenger RNA [mRNA]), was observed in the brain tissue of MS patients, especially in acute and chronic active lesions, compared to healthy controls [48]. Th17 cells may also have a role in progressive forms of MS [49]. Finally, levels of GM-CSF, which is essential for Th17 responses as discussed earlier in this review, were also shown to be elevated in the CSF of MS patients $[50,51]$ and in the blood of MS (but not of NMO) patients [52].

Th17-related molecules were shown to correlate with parameters of disease activity in MS. In vitro studies demonstrated that the amount of IL-17 (and also IL-5) produced by mononuclear cell cultures from patients with MS after stimulation with human myelin basic protein correlates with the number of active lesions on magnetic resonance imaging (MRI) [53]. The proportion of Th17 cells, their subset effector memory Th17 cells (CD4+/CD45RO+/CCR7-), and the level of IL-17A correlated with disease severity as measured by the Expanded Disability Status Scale (EDSS), while the proportion of another subset, central memory Th17 cells (CD4+/CD45RO+/CCR7+), correlated with relapse frequency, in both MS and NMO [54]. Serum IL-17F (but not IL$17 \mathrm{~A})$ correlated with the number of MS relapses in two years [47].

\section{Th17 in NMOSD and Its Animal Models}

Much of the evidence regarding pathogenesis of NMOSD comes from studies on opticospinal MS (OSMS) and NMO. The former is no longer considered to be a variant of MS, since most of those patients actually had NMO [3]; hence OSMS is considered as an obsolete term.

Although the important role of B cell autoimmunity against aquaporin-4 (AQP4), by means of the anti-AQP4 immunoglobulin G (IgG), a T cell dependent immunoglobulin type (IgG1), in mediating CNS lesions is clearly established, many aspects of tissue damage in NMOSD remain poorly understood [55]. However, T cell-related mechanisms have been increasingly implicated in NMOSD [56-58]. AQP4-specific T cell responses were demonstrated to be amplified in NMO patients, whose T cells were shown to exhibit a Th17 polarization, partially mediated by increased production of IL-6 [59].

In some studies, IL-17 was increased in the CSF [60, 61] and in the blood [54] of NMO patients. Another study on cytokines in NMO did not find an increase in serum or CSF levels of IL-17 but did find an increased level of other Th17-related cytokines, notably IL-6 [52], which is a proinflammatory cytokine that increases the survival of plasmablasts capable of producing anti-AQP4-IgG and is also involved in the development of Th17 cells, which can also support B cell development and induce further tissue injury [62].

Th17-related markers have also been shown to correlate with parameters of disease activity and severity in NMOSD. The release of IL- 6 and IL- 21 by polyclonally activated CD4+ $\mathrm{T}$ cells derived from NMO patients was demonstrated to correlate directly with neurological disability [63] and in vivo and in vitro levels of IL-6 were higher among NMO patients who experienced relapse within a 2-year follow-up [64]. CSF levels of both IL-17A and the downstream cytokine IL-8 were found to have a positive correlation with spinal cord lesion length in NMO [60]. As previously mentioned in studies with MS, the proportion of effector memory Th17 cells and IL-17A levels correlated with EDSS, and the proportion of central memory Th17 cells correlated with relapse frequency in NMO [54].

The development of NMO-like disease models in animals has provided important insights into the pathogenesis of NMOSD. After the pathogenic potential of anti-AQP4-IgG has been demonstrated, it was shown that AQP4-specific T cells could also induce a NMO-like disease in rats, independent of anti-AQP4-IgG $[65,66]$. Recently, another NMO-like model was developed in mice using AQP4-reactive T cells polarized into a Th17 phenotype (also independent of antiAQP4-IgG), promoting lesions in the optic nerve and spinal cord [67].

A summary of the main studies supporting the involvement of Th17-related pathways in NMOSD is provided in Table 2.

\section{Different Immunologic Profiles in MS and NMOSD}

Several studies have already addressed the immunological differences between MS and NMO. However, a clear definition of immunologic profiles that differentiate MS and NMO remains controversial.

Investigators have assessed the levels of several cytokines and chemokines in the serum and/or CSF of patients with NMO and MS and compared them between both diseases and between each of them and a control group composed of patients with noninflammatory neurological disorders [52]. Both Th2- and Th17-related molecules were found to be 
TABLE 2: Hallmarks on the understanding of the role of the Th17 pathways in NMOSD.

\begin{tabular}{lc}
\hline Finding & Reference \\
\hline Increased IL-17 found in the CSF of NMO patients & {$[60]$} \\
\hline $\begin{array}{l}\text { Increased Th17 cells found in the blood of NMO } \\
\text { patients, especially during relapse }\end{array}$ & {$[69]$} \\
\hline $\begin{array}{l}\text { IL-17 and subsets of Th17 correlate with EDSS and } \\
\text { relapse frequency in NMO }\end{array}$ & {$[54]$} \\
$\begin{array}{l}\text { Pathogenicity of AQP4-specific T cells demonstrated in } \\
\text { animal models }\end{array}$ & {$[65]$} \\
\hline $\begin{array}{l}\text { Pathogenicity of Th17-polarized AQP4-specific T cells } \\
\text { demonstrated in animal models }\end{array}$ & {$[67]$} \\
\hline
\end{tabular}

upregulated in NMO, except, interestingly, for the signature cytokine of each of those pathways, that is, IL-4 and IL17 family [52]. Of note, among the Th17-related molecules elevated in NMO, IL-6 seemed to be the most relevant one [52]. In the same study, the Treg-related cytokine IL-10 was elevated in both NMO and MS, whereas Th1-related cytokines and molecules were upregulated only in MS [52].

Another study found increased levels of Th1-related markers in NMO when compared to MS, while the levels of Th17-related markers were similar between both diseases [68]. Investigators from the same group found increased proportion of Th17 cells and of IL-17-secreting T CD8+ cells, in both MS and NMO, especially during relapses [69]. The same study did find IL-17 levels, as well as those of IL-23, to be higher in NMO than in MS, leading the authors to speculate whether that would explain the more aggressive nature of NMO when compared to MS [69].

Another study compared NMO, RRMS, and PPMS and reported an increased expression of Th17- and Th1-related cytokines as being characteristic of NMO [70]. Further studies are required to clarify if cytokine levels are useful to indicate disease activity and if interference in the Th17 pathway can reduce inflammation in the CNS during relapses.

\section{Effects of Current Therapy on the Th17 Axis}

Since Th17 responses seem to be relevant in the pathogenesis of MS and NMOSD, great interest has been put in identifying possible effects of the currently available therapeutic agents on the Th17 pathways. A better understanding of those effects and of the different immunologic profiles of MS and NMOSD could potentially provide an explanation to why some NMOSD patients get worse when undergoing treatment with MS-targeted disease-modifying therapies, such as interferons. It could also provide valuable insights into potential mechanisms to be addressed by new or repositioned drugs.

Intravenous methylprednisolone (IVMP) is the most widely used treatment for acute relapses in both MS and NMOSD. A significant reduction in Th17 cell counts, IL-17A and IL-23R production, and RAR-related orphan receptor $\mathrm{c}$ (RORc) mRNA expression has been observed in MS patients after IVMP pulse therapy $[54,71]$. A reduction in the same markers, except for IL-17A, was also seen in NMO patients after IVMP treatment [54]. Even though Th17 cells as a whole were decreased in both NMO and MS after IVMP therapy, a stratified assessment of their responses according to some of their subsets showed that central memory Th17 and effector memory Th17 cells were decreased only in NMO and not in MS [54]. These differences in the response to corticosteroids may explain the effectiveness of such drugs in NMO to reduce the risk of further attacks [54]. Progressive MS patients undergoing monthly IVMP pulse therapy presented no changes in the phenotype of Th17 cells [72].

Another therapeutic option for acute relapses in MS and NMOSD is intravenous immunoglobulin (IVIg). In vitro studies have demonstrated that IVIg inhibits the differentiation and amplification of Th17 cells and the production of IL-17A, IL-17F, IL-21, and CCL20 [73]. The inhibitory effect of IVIg on IL-17A seems to be mediated by the modulation of intracellular signaling pathways and not by passive neutralization by anti-IL-17 antibodies from the IVIg preparations [74].

Several drugs have been developed as disease-modifying therapies for MS, and many of them have been shown to modulate the Th17 axis. One of these immunomodulatory drugs is the recombinant IFN- $\beta$, which includes IFN- $\beta$ la and IFN- $\beta$ 1b. It was suggested that IFN- $\beta$ inhibits the differentiation of Th17 cells in mice and hence modulates the severity of EAE, by acting on the toll-IL-1 receptor domain-containing adaptor inducing IFN- $\beta$-dependent (TRIF-dependent) type I IFN induction pathway and its downstream signaling pathways, especially by means of an increased production of IL-27 [75]. In both mice with EAE and patients with MS, IFN- $\beta$ also decreased the ability of dendritic cells to stimulate the production of IL-17 by Th17 cells and increased the production of IL-27 by dendritic cells, shifting the proinflammatory response into an antiinflammatory one [76]. In humans, IFN- $\beta$ therapy was shown to downregulate the expression of IL- $1 \beta$, IL-23R, RORc, and IL-17A and upregulate the expression of IL-12, IL-27, and IL-10, suppressing the differentiation of näive $\mathrm{T}$ cells into Th17 cells. These effects may explain some of the IFN- $\beta$ 's immunomodulatory effect in MS [77].

However, IFN- $\beta$ is not always effective in reducing CNS autoimmunity, and efforts have been done to identify factors that could predict the response to IFN- $\beta$ therapy. One study pointed out that mice with Th1-induced EAE did benefit from IFN- $\beta$ treatment, whereas deleterious effects were observed in mice with Th17-induced EAE [78]. Indeed, IFN- $\beta$ seems to be effective in diseases primarily driven by Th1, whereas it has proinflammatory effects in Th2-driven diseases [79]. In a study with RRMS patients, pretreatment levels of IL$17 \mathrm{~F}$ and of endogenous IFN- $\beta$ were higher in nonresponders than in IFN- $\beta$ treatment responders [78]. A subsequent study, however, did not confirm the role of serum IL-17F in the prediction of poor response to IFN- $\beta$ therapy [80]. Interestingly, IFN- $\beta$ is not effective in reducing relapse rates or preventing disability in patients with NMOSD [81] and may even trigger severe exacerbations in those patients [82, 83]. It has been suggested that the poor response to IFN- $\beta$ in NMOSD may be related to elevated levels of IL-17 [4], which 
corroborates some of the findings from earlier studies with EAE models and MS patients.

Glatiramer acetate (GA) is another traditional diseasemodifying drug for MS. In EAE mice treated with GA, Th17 cells were drastically reduced, while Treg cells were increased [84]. However, further studies are required to clarify if the same effects are observed in MS patients treated with GA. Despite some anecdotal reports of patients with NMOSD who seemed to benefit from GA therapy [85, 86], as well as some speculations regarding the rationale for a potential benefit of GA in NMOSD [87], currently there is no evidence to support this indication, as well as no data regarding its effect on Th17 in NMOSD patients.

In patients with MS, fingolimod (FTY720) reduces Th17 central memory $\mathrm{T}$ cells in peripheral blood, presumably due to the retention of those cells in secondary lymphoid organs [88]. Another study pointed out that, after initiation of fingolimod therapy, half of the patients had a reduction in the proportion of circulating Th17 cells, whereas the other half (including the only one patient with relapses in that sample during the follow-up period) had an increase in the proportion of those cells, suggesting that a slower reduction in circulating Th17 cells after fingolimod initiation would predispose to relapses [89]. In NMOSD, however, treatment with fingolimod was reported to trigger extensive brain lesions [90] or a fulminant course [91]; thus it is not recommended for NMOSD. Since eosinophils have been implicated in NMOSD pathogenesis [92] and fingolimod may promote bone marrow egress of eosinophils [93] and other pathogenic proinflammatory cells, that would explain the severe disease activity in NMOSD patients exposed to fingolimod.

Natalizumab is another commonly used treatment for MS. It acts by interfering with lymphocyte migration across the $\mathrm{BBB}$, which is mediated by the interaction between $\alpha 4 \beta 1$ integrin (on the surface of lymphocytes) and vascular-cell adhesion molecule 1 (VCAM-1; an endothelial receptor in CNS vessels) [94]. Specifically, it binds to the integrins, thus preventing them from binding to their endothelial receptors [94]. Even though such process is also required for CNS inflammation to develop in NMOSD, natalizumab was reported to fail in controlling disease activity in patients with NMOSD [95] or even triggering catastrophic exacerbations $[96,97]$. Like fingolimod, natalizumab may increase the number of peripheral eosinophils [94], which could account for the increased disease activity in NMOSD patients treated with natalizumab. The specific effects of natalizumab over the Th17 cells have not been described, and given the number of receptors present in such cells, it might be possible that pathogenic Th17 cells can use alternative pathways not requiring binding to $\alpha 4 \beta 1$ integrin to access the CNS.

In MS patients, treatment with dimethyl fumarate inhibited the maturation of dendritic cells and thus the generation of IFN- $\gamma$-producing (Th1) and IL-17-producing (Th17) cells [98]. No evidence on the use of dimethyl fumarate in NMOSD is available, so the use of this drug is not recommended at this time point.

Finally, treatment of aggressive MS with chemoablation and hematopoietic stem cell transplantation seems to exert its effect by decreased Th17 and Th1/Th17 responses, rather than Th1 pathway responses [99]. Even though some studies reported cases of NMOSD treated with stem cell transplantation [100-103], with variable results, none of them reported its influence on Th17 cells.

Several cytotoxic, immunomodulatory, and B cell depleting therapies are available for prevention of attacks in NMOSD, including prophylactic corticosteroids, plasma exchange, and IVIg, as well as azathioprine, mycophenolate mofetil, methotrexate, mitoxantrone, cyclophosphamide, and rituximab [104]; however, their precise effects on Thl7 cells in NMOSD are not known to date. Rituximab (an anti-CD20 monoclonal antibody) and IVIg in combination have been shown to modulate $\mathrm{T}$ cell subsets and humoral immune responses in NMOSD [105]. Some data regarding the specific effects of the aforementioned drugs on the Th17 pathways is available from studies on other autoimmune diseases. Rituximab decreases Th17 cell responses in rheumatoid arthritis [106] and the IL-17 production in Sjögren's syndrome [107]. Methotrexate, with or without corticosteroids, reduces Th17 cell frequency [108], normalizes the Th17/Treg balance [109], and suppresses IL-17 production [110] in rheumatoid arthritis.

\section{Emerging Therapies Targeting the Th17 Pathways}

A few monoclonal antibodies targeting different Thl7-related cytokines have been tested in MS so far. The first one, ustekinumab, was an antibody against IL12 and IL-23, which are critical for the maintenance of Th17 cells. Nevertheless, in a phase II study, ustekinumab did not show efficacy in the reduction of new enhancing lesions on brain MRI, number of relapses, or change from baseline EDSS after 23 weeks [111].

Secukinumab (AIN457) is a recombinant, highly selective, fully human monoclonal antibody against IL-17A. In vitro treatment of human astrocytes with secukinumab was shown to upregulate the levels of IL-6 and to decrease the levels of proinflammatory molecules [112], thus making it suitable for phase II studies in MS. Clinical trials in some other autoimmune diseases yielded promising results, with no significant safety concerns [113], and so far, three phase II trials assessing secukinumab in MS have been started. The first one (ClinicalTrials.gov identifier: NCT01051817) was a randomized, multicenter, double-blind, proof-of-concept study to assess the effect of secukinumab versus placebo on MRI parameters of disease activity over a 24 -week period in patients with RRMS [114]. The results have been presented in a conference, as follows: the primary outcomes, number of new gadolinium-enhancing lesions, and number of combined unique active lesions decreased by $67 \%(P=0.003)$ and by $49 \%(P=0.087)$, respectively, in the secukinumab group; as a secondary outcome, the annualized relapse rate decreased by $43 \%$ in the secukinumab group, which was not statistically significant, maybe because the study was not powered to assess that outcome [114]. However, due to methodological issues (especially small sample size and important demographic, clinical, and radiological differences between groups on baseline), caution must be taken in the 
interpretation of those findings [115]. The second trial (ClinicalTrials.gov identifier: NCT01874340), which is an extension of the first one, has had no results published yet. The third trial (ClinicalTrials.gov identifier: NCT01874340), which is a larger phase II study, was terminated early "based upon development of another anti-IL-17 fully human monoclonal antibody with better potential for treating MS patients," according to information provided by the sponsor. Actually, the new compound is CJM112, a new fully human antiIL17 A monoclonal antibody, whose phase II trial was not yet registered on https://ClinicalTrials.gov (accessed on 19 September 2015).

Future perspectives include targeting genes and soluble factors that mediate Th17 cell expansion and have been shown to be differentially expressed in MS patients [116], as well as modulating metabolic pathways that are relevant for the regulation of Th17 responses [30, 31].

\section{Conclusions}

Even though not completely understood, the role of Th17 cells in the pathogenesis of both MS and NMOSD is very well established by several findings from studies in humans and animal models. Th17-related pathways seem to be modulated by many of the currently available therapies, and drugs targeting specific points on those pathways are already being tested on phase II studies, with promising results. Further studies focusing on the role of Th17 cells and their related molecules as biomarkers of diagnosis, disease activity, and response to specific therapies are warranted and may potentially lead to a more precise comprehension of MS and NMOSD, as well as more selective and effective therapies.

\section{Disclaimer}

The funding sources had no role in the design, collection, analysis, or interpretation of the study nor in the writing of the paper or decision to submit.

\section{Conflict of Interests}

Dr. Passos reports no conflict of interests; Dr. Sato has received scholarship from the Ministry of Education, Culture, Sports, Science and Technology (MEXT) of Japan, Grantin-Aid for scientific research from the Japan Society for the Promotion of Science (KAKENHI 15K19472), research support from CAPES/Brasil (CSF-PAJT-88887.091277/201400 ), and speaker honoraria from Novartis and Genzyme; Dr. Becker reports travel support, research grants, and speaker honoraria from Bayer Healthcare, Biogen Idec, Genzyme, Merck Serono, Novartis, Roche, and Teva; Dr. Fujihara serves on Scientific Advisory Boards for Bayer Schering Pharma, Biogen Idec, Mitsubishi Tanabe Pharma Corporation, Novartis Pharma, Chugai Pharmaceutical, Ono Pharmaceutical, Nihon Pharmaceutical, Merck Serono, Alexion Pharmaceuticals, Medimmune, and Medical Review; has received funding for travel and speaker honoraria from Bayer Schering Pharma, Biogen Idec, Eisai Inc., Mitsubishi Tanabe Pharma
Corporation, Novartis Pharma, Astellas Pharma Inc., Takeda Pharmaceutical Company Limited, Asahi Kasei Medical Co., Daiichi Sankyo, and Nihon Pharmaceutical; serves as an Editorial Board Member of Clinical and Experimental Neuroimmunology (2009-present) and an Advisory Board Member of Sri Lanka Journal of Neurology; has received research support from Bayer Schering Pharma, Biogen Idec Japan, Asahi Kasei Medical, the Chemo-Sero-Therapeutic Research Institute, Teva Pharmaceutical, Mitsubishi Tanabe Pharma, Teijin Pharma, Chugai Pharmaceutical, Ono Pharmaceutical, Nihon Pharmaceutical, and Genzyme Japan; and is funded as the Secondary Investigator (\#22229008, 2010-2015) by the Grants-in-Aid for Scientific Research from the Ministry of Education, Science and Technology of Japan and as the Secondary Investigator by the Grants-in-Aid for Scientific Research from the Ministry of Health, Welfare and Labor of Japan (2010-present).

\section{Acknowledgments}

This study was partially supported by Grant-in-Aid for scientific research from the Japan Society for the Promotion of Science (KAKENHI 15K19472) and CAPES/Brasil (CSFPAJT- 88887.091277/2014-00).

\section{References}

[1] A. Compston and A. Coles, "Multiple sclerosis," The Lancet, vol. 372, no. 9648, pp. 1502-1517, 2008.

[2] K. Fujihara, T. Misu, I. Nakashima et al., "Neuromyelitis optica should be classified as an astrocytopathic disease rather than a demyelinating disease," Clinical and Experimental Neuroimmunology, vol. 3, no. 2, pp. 58-73, 2012.

[3] D. M. Wingerchuk, B. Banwell, J. L. Bennett et al., "International consensus diagnostic criteria for neuromyelitis optica spectrum disorders," Neurology, vol. 85, no. 2, pp. 177-189, 2015.

[4] K.-C. Wang, K.-H. Lin, T.-C. Lee et al., "Poor responses to interferon-beta treatment in patients with neuromyelitis optica and multiple sclerosis with long spinal cord lesions," PLOS ONE, vol. 9, no. 6, Article ID e98192, 2014.

[5] T. R. Mosmann, H. Cherwinski, M. W. Bond, M. A. Giedlin, and R. L. Coffman, "Two types of murine helper T cell clone. I. Definition according to profiles of lymphokine activities and secreted proteins," The Journal of Immunology, vol. 136, no. 7, pp. 2348-2357, 1986.

[6] A. Rostami and B. Ciric, "Role of Th17 cells in the pathogenesis of CNS inflammatory demyelination," Journal of the Neurological Sciences, vol. 333, no. 1-2, pp. 76-87, 2013.

[7] C. Sie, T. Korn, and M. Mitsdoerffer, "Th17 cells in central nervous system autoimmunity," Experimental Neurology, vol. 262, part A, pp. 18-27, 2014.

[8] L. E. Harrington, R. D. Hatton, P. R. Mangan et al., "Interleukin 17-producing $\mathrm{CD} 4^{+}$effector T cells develop via a lineage distinct from the T helper type 1 and 2 lineages," Nature Immunology, vol. 6, no. 11, pp. 1123-1132, 2005.

[9] H. Park, Z. Li, X. O. Yang et al., "A distinct lineage of CD4 T cells regulates tissue inflammation by producing interleukin 17," Nature Immunology, vol. 6, no. 11, pp. 1133-1141, 2005.

[10] C. L. Langrish, Y. Chen, W. M. Blumenschein et al., "IL-23 drives a pathogenic $\mathrm{T}$ cell population that induces autoimmune 
inflammation," The Journal of Experimental Medicine, vol. 201, no. 2, pp. 233-240, 2005.

[11] D. J. Cua, J. Sherlock, Y. Chen et al., "Interleukin-23 rather than interleukin-12 is the critical cytokine for autoimmune inflammation of the brain," Nature, vol. 421, no. 6924, pp. 744-748, 2003.

[12] K. Ghoreschi, A. Laurence, X.-P. Yang et al., "Generation of pathogenic $\mathrm{T}_{H} 17$ cells in the absence of TGF- $\beta$ signalling," Nature, vol. 467, no. 7318, pp. 967-971, 2010.

[13] D. W. Luchtman, E. Ellwardt, C. Larochelle, and F. Zipp, "IL-17 and related cytokines involved in the pathology and immunotherapy of multiple sclerosis: current and future developments," Cytokine and Growth Factor Reviews, vol. 25, no. 4, pp. 403-413, 2014.

[14] K. Hirota, J. H. Duarte, M. Veldhoen et al., "Fate mapping of IL-17-producing T cells in inflammatory responses," Nature Immunology, vol. 12, no. 3, pp. 255-263, 2011.

[15] H. Kebir, I. Ifergan, J. I. Alvarez et al., "Preferential recruitment of interferon- $\gamma$-expressing TH17 cells in multiple sclerosis," Annals of Neurology, vol. 66, no. 3, pp. 390-402, 2009.

[16] M. El-Behi, B. Ciric, H. Dai et al., "The encephalitogenicity of $\mathrm{T}(\mathrm{H}) 17$ cells is dependent on IL-1- and IL-23-induced production of the cytokine GM-CSF," Nature Immunology, vol. 12, no. 6, pp. 568-575, 2011.

[17] I. Sonderegger, G. Iezzi, R. Maier, N. Schmitz, M. Kurrer, and M. Kopf, "GM-CSF mediates autoimmunity by enhancing IL6-dependent Th17 cell development and survival," Journal of Experimental Medicine, vol. 205, no. 10, pp. 2281-2294, 2008.

[18] J. L. McQualter, R. Darwiche, C. Ewing et al., "Granulocyte macrophage colony-stimulating factor: a new putative therapeutic target in multiple sclerosis," Journal of Experimental Medicine, vol. 194, no. 7, pp. 873-882, 2001.

[19] L. Codarri, G. Gyülvészi, V. Tosevski et al., "ROR $\gamma$ t drives production of the cytokine GM-CSF in helper T cells, which is essential for the effector phase of autoimmune neuroinflammation," Nature Immunology, vol. 12, no. 6, pp. 560-567, 2011.

[20] W. Sheng, F. Yang, Y. Zhou et al., "STAT5 programs a distinct subset of GM-CSF-producing T helper cells that is essential for autoimmune neuroinflammation," Cell Research, vol. 24, no. 12, pp. 1387-1402, 2014.

[21] Y. Komiyama, S. Nakae, T. Matsuki et al., "IL-17 plays an important role in the development of experimental autoimmune encephalomyelitis," Journal of Immunology, vol. 177, no. 1, pp. 566-573, 2006.

[22] X. O. Yang, S. H. Chang, H. Park et al., "Regulation of inflammatory responses by IL-17F," The Journal of Experimental Medicine, vol. 205, no. 5, pp. 1063-1075, 2008.

[23] H. S. Domingues, M. Mues, H. Lassmann, H. Wekerle, and G. Krishnamoorthy, "Functional and pathogenic differences of Th1 and Th17 cells in experimental autoimmune encephalomyelitis," PLoS ONE, vol. 5, no. 11, Article ID e15531, 2010.

[24] F. Odoardi, C. Sie, K. Streyl et al., "T cells become licensed in the lung to enter the central nervous system," Nature, vol. 488, no. 7413, pp. 675-679, 2012.

[25] H. Wekerle, K. Berer, and G. Krishnamoorthy, "Remote controltriggering of brain autoimmune disease in the gut," Current Opinion in Immunology, vol. 25, no. 6, pp. 683-689, 2013.

[26] F. J. Quintana, A. S. Basso, A. H. Iglesias et al., "Control of $\mathrm{T}(\mathrm{reg})$ and $\mathrm{T}(\mathrm{H}) 17$ cell differentiation by the aryl hydrocarbon receptor," Nature, vol. 453, pp. 65-71, 2008.
[27] I. I. Ivanov, K. Atarashi, N. Manel et al., "Induction of intestinal Th17 cells by segmented filamentous bacteria," Cell, vol. 139, no. 3, pp. 485-498, 2009.

[28] V. Gaboriau-Routhiau, S. Rakotobe, E. Lécuyer et al., "The key role of segmented filamentous bacteria in the coordinated maturation of gut helper T cell responses," Immunity, vol. 31, no. 4, pp. 677-689, 2009.

[29] P. O. Barros, U. C. Linhares, B. Teixeira et al., "High in vitro immune reactivity to Escherichia coli in neuromyelitis optica patients is correlated with both neurological disabilities and elevated plasma lipopolysaccharide levels," Human Immunology, vol. 74, no. 9, pp. 1080-1087, 2013.

[30] J. Barbi, D. Pardoll, and F. Pan, "Metabolic control of the Treg/Th17 axis," Immunological Reviews, vol. 252, no. 1, pp. 5277, 2013.

[31] K. Yang and H. Chi, "Metabolic control of Th17 cell generation and CNS inflammation," Journal of Neurology \& Neurophysiology, vol. S12, article 004, 2014.

[32] M. Kleinewietfeld, A. Manzel, J. Titze et al., "Sodium chloride drives autoimmune disease by the induction of pathogenic TH17 cells," Nature, vol. 496, no. 7446, pp. 518-522, 2013.

[33] C. Wu, N. Yosef, T. Thalhamer et al., "Induction of pathogenic TH 17 cells by inducible salt-sensing kinase SGK1," Nature, vol. 496, no. 7446, pp. 513-517, 2013.

[34] M. F. Farez, F. J. Quintana, and J. Correale, "Sodium intake is associated with increased disease activity in multiple sclerosis," Journal of Neurology, Neurosurgery \& Psychiatry, vol. 86, no. 1, pp. 26-31, 2015.

[35] P. O. Barros, T. B. Ferreira, M. M. M. Vieira et al., "Substance P enhances Thl7 phenotype in individuals with generalized anxiety disorder: an event resistant to glucocorticoid inhibition," Journal of Clinical Immunology, vol. 31, no. 1, pp. 51-59, 2011.

[36] H. Kebir, K. Kreymborg, I. Ifergan et al., "Human TH17 lymphocytes promote blood-brain barrier disruption and central nervous system inflammation," Nature Medicine, vol. 13, no. 10, pp. 1173-1175, 2007.

[37] T. Carlson, M. Kroenke, P. Rao, T. E. Lane, and B. Segal, "The Th17-ELR+ CXC chemokine pathway is essential for the development of central nervous system autoimmune disease," The Journal of Experimental Medicine, vol. 205, no. 4, pp. 811823, 2008.

[38] A. Reboldi, C. Coisne, D. Baumjohann et al., "C-C chemokine receptor 6-regulated entry of $\mathrm{T}_{\mathrm{H}}-17$ cells into the CNS through the choroid plexus is required for the initiation of EAE," Nature Immunology, vol. 10, no. 5, pp. 514-523, 2009.

[39] J. Huppert, D. Closhen, A. Croxford et al., "Cellular mechanisms of IL-17-induced blood-brain barrier disruption," The FASEB Journal, vol. 24, no. 4, pp. 1023-1034, 2010.

[40] V. Rothhammer, S. Heink, F. Petermann et al., "Th17 lymphocytes traffic to the central nervous system independently of $\alpha 4$ integrin expression during EAE," The Journal of Experimental Medicine, vol. 208, no. 12, pp. 2465-2476, 2011.

[41] T. Schneider-Hohendorf, J. Rossaint, H. Mohan et al., "VLA4 blockade promotes differential routes into human CNS involving PSGL-1 rolling of T cells and MCAM-adhesion of TH17 cells," Journal of Experimental Medicine, vol. 211, no. 9, pp. 1833-1846, 2014.

[42] Á. C. Murphy, S. J. Lalor, M. A. Lynch, and K. H. G. Mills, "Infiltration of Th1 and Th17 cells and activation of microglia in the CNS during the course of experimental autoimmune encephalomyelitis," Brain, Behavior, and Immunity, vol. 24, no. 4, pp. 641-651, 2010. 
[43] D. Matusevicius, P. Kivisäkk, B. He et al., "Interleukin-17 mRNA expression in blood and CSF mononuclear cells is augmented in multiple sclerosis," Multiple Sclerosis, vol. 5, no. 2, pp. 101-104, 1999.

[44] V. Brucklacher-Waldert, K. Stuerner, M. Kolster, J. Wolthausen, and E. Tolosa, "Phenotypical and functional characterization of T helper 17 cells in multiple sclerosis," Brain, vol. 132, no. 12, pp. 3329-3341, 2009.

[45] L. Durelli, L. Conti, M. Clerico et al., "T-helper 17 cells expand in multiple sclerosis and are inhibited by interferon- $\beta$," Annals of Neurology, vol. 65, no. 5, pp. 499-509, 2009.

[46] Z. Babaloo, F. Babaie, M. Farhoodi et al., "Interleukin-17A and interleukin-17F mRNA expressions in peripheral blood mononuclear cells of patients with multiple sclerosis," Iranian Journal of Immunology, vol. 7, no. 4, pp. 202-209, 2010.

[47] Z. Babaloo, M. R. Aliparasti, F. Babaiea, S. Almasi, B. Baradaran, and M. Farhoudi, "The role of Th17 cells in patients with relapsing-remitting multiple sclerosis: interleukin-17A and interleukin-17F serum levels," Immunology Letters, vol. 164, no. 2, pp. 76-80, 2015.

[48] J. S. Tzartos, M. A. Friese, M. J. Craner et al., "Interleukin-17 production in central nervous system-infiltrating $\mathrm{T}$ cells and glial cells is associated with active disease in multiple sclerosis," The American Journal of Pathology, vol. 172, no. 1, pp. 146-155, 2008.

[49] J. R. Christensen, L. Börnsen, R. Ratzer et al., "Systemic inflammation in progressive multiple sclerosis involves follicular T-helper, Th17- and activated B-cells and correlates with progression," PLoS ONE, vol. 8, no. 3, Article ID e57820, 2013.

[50] O. Perrella, P. B. Carrieri, R. De Mercato, and G. A. Buscaino, "Markers of activated $\mathrm{T}$ lymphocytes and $\mathrm{T}$ cell receptor gamma/delta+ in patients with multiple sclerosis," European Neurology, vol. 33, no. 2, pp. 152-155, 1993.

[51] P. B. Carrieri, V. Provitera, T. De Rosa, G. Tartaglia, F. Gorga, and O. Perrella, "Profile of cerebrospinal fluid and serum cytokines in patients with relapsing-remitting multiple sclerosis: a correlation with clinical activity," Immunopharmacology and Immunotoxicology, vol. 20, no. 3, pp. 373-382, 1998.

[52] A. Uzawa, M. Mori, K. Arai et al., "Cytokine and chemokine profiles in neuromyelitis optica: significance of interleukin-6," Multiple Sclerosis, vol. 16, no. 12, pp. 1443-1452, 2010.

[53] C. J. Hedegaard, M. Krakauer, K. Bendtzen, H. Lund, F. Sellebjerg, and C. H. Nielsen, "T helper cell type 1 (Th1), Th2 and Th17 responses to myelin basic protein and disease activity in multiple sclerosis," Immunology, vol. 125, no. 2, pp. 161-169, 2008.

[54] Y. Li, H. Wang, Y. Long, Z. Lu, and X. Hu, "Increased memory Th17 cells in patients with neuromyelitis optica and multiple sclerosis," Journal of Neuroimmunology, vol. 234, no. 1-2, pp. 155-160, 2011.

[55] P. A. Nelson, M. Khodadoust, T. Prodhomme et al., "Immunodominant $\mathrm{T}$ cell determinants of aquaporin-4, the autoantigen associated with neuromyelitis optica," PLoS ONE, vol. 5, no. 11, Article ID e15050, 2010.

[56] N. Matsuya, M. Komori, K. Nomura et al., "Increased Tcell immunity against aquaporin-4 and proteolipid protein in neuromyelitis optica," International Immunology, vol. 23, no. 9, pp. 565-573, 2011.

[57] W. Bukhari, M. H. Barnett, K. Prain, and S. A. Broadley, "Molecular pathogenesis of neuromyelitis optica," International Journal of Molecular Sciences, vol. 13, no. 10, pp. 12970-12993, 2012.
[58] M. Mitsdoerffer, V. Kuchroo, and T. Korn, "Immunology of neuromyelitis optica: a T cell-B cell collaboration," Annals of the New York Academy of Sciences, vol. 1283, no. 1, pp. 57-66, 2013.

[59] M. Varrin-Doyer, C. M. Spencer, U. Schulze-Topphoff et al., "Aquaporin 4-specific T cells in neuromyelitis optica exhibit a Th17 bias and recognize Clostridium ABC transporter," Annals of Neurology, vol. 72, no. 1, pp. 53-64, 2012.

[60] T. Ishizu, M. Osoegawa, F.-J. Mei et al., "Intrathecal activation of the IL-17/IL-8 axis in opticospinal multiple sclerosis," Brain, vol. 128, no. 5, pp. 988-1002, 2005.

[61] M. Tanaka, T. Matsushita, T. Tateishi et al., "Distinct CSF cytokine/chemokine profiles in atopic myelitis and other causes of myelitis," Neurology, vol. 71, no. 13, pp. 974-981, 2008.

[62] A. Uzawa, M. Mori, and S. Kuwabara, "Neuromyelitis optica: concept, immunology and treatment," Journal of Clinical Neuroscience, vol. 21, no. 1, pp. 12-21, 2014.

[63] U. C. Linhares, P. B. Schiavoni, P. O. Barros et al., "The ex vivo production of IL- 6 and IL- 21 by $\mathrm{CD}^{+}{ }^{+} \mathrm{T}$ cells is directly associated with neurological disability in neuromyelitis optica patients," Journal of Clinical Immunology, vol. 33, no. 1, pp. 179189, 2013.

[64] P. O. Barros, T. Cassano, J. Hygino et al., "Prediction of disease severity in neuromyelitis optica by the levels of interleukin (IL)6 produced during remission phase," Clinical \& Experimental Immunology, 2015.

[65] M. Pohl, M. T. Fischer, S. Mader et al., "Pathogenic T cell responses against aquaporin 4," Acta Neuropathologica, vol. 122, no. 1, pp. 21-34, 2011.

[66] M. Bradl and H. Lassmann, "Experimental models of neuromyelitis optica," Brain Pathology, vol. 24, no. 1, pp. 74-82, 2014.

[67] M. V. Jones, H. Huang, P. A. Calabresi, and M. Levy, "Pathogenic aquaporin- 4 reactive $\mathrm{T}$ cells are sufficient to induce mouse model of neuromyelitis optica," Acta Neuropathologica Communications, vol. 3, no. 1, article 28, 2015.

[68] K. C. Wang, C.-L. Lee, S.-Y. Chen et al., "Distinct serum cytokine profiles in neuromyelitis optica and multiple sclerosis," Journal of Interferon and Cytokine Research, vol. 33, no. 2, pp. 58-64, 2013.

[69] H. H. Wang, Y. Q. Dai, W. Qiu et al., "Interleukin-17-secreting $\mathrm{T}$ cells in neuromyelitis optica and multiple sclerosis during relapse," Journal of Clinical Neuroscience, vol. 18, no. 10, pp. 13131317, 2011.

[70] T. Matsushita, T. Tateishi, N. Isobe et al., "Characteristic cerebrospinal fluid cytokine/chemokine profiles in neuromyelitis optica, relapsing remitting or primary progressive multiple sclerosis," PLoS ONE, vol. 8, no. 4, Article ID e61835, 2013.

[71] M. Liu, X. Hu, Y. Wang et al., "Effect of high-dose methylprednisolone treatment on Th17 cells in patients with multiple sclerosis in relapse," Acta Neurologica Scandinavica, vol. 120, no. 4, pp. 235-241, 2009.

[72] R. Ratzer, J. Romme Christensen, B. Romme Nielsen, P. S. Sørensen, L. Börnsen, and F. Sellebjerg, "Immunological effects of methylprednisolone pulse treatment in progressive multiple sclerosis," Journal of Neuroimmunology, vol. 276, no. 1-2, pp. 195-201, 2014.

[73] M. S. Maddur, J. Vani, P. Hegde, S. Lacroix-Desmazes, S. V. Kaveri, and J. Bayry, "Inhibition of differentiation, amplification, and function of human T H17 cells by intravenous immunoglobulin," Journal of Allergy and Clinical Immunology, vol. 127, no. 3, pp. 823-830, 2011. 
[74] M. S. Maddur, M. Sharma, P. Hegde, S. Lacroix-Desmazes, S. V. Kaveri, and J. Bayry, "Inhibitory effect of IVIG on IL-17 production by th17 cells is independent of Anti-IL-17 antibodies in the immunoglobulin preparations," Journal of Clinical Immunology, vol. 33, supplement 1, pp. 62-66, 2013.

[75] B. Guo, E. Y. Chang, and G. Cheng, "The type I IFN induction pathway constrains Th17-mediated autoimmune inflammation in mice," The Journal of Clinical Investigation, vol. 118, no. 5, pp. 1680-1690, 2008.

[76] C. M. Sweeney, R. Lonergan, S. A. Basdeo et al., "IL-27 mediates the response to IFN- $\beta$ therapy in multiple sclerosis patients by inhibiting Th17 cells," Brain, Behavior, and Immunity, vol. 25, no. 6, pp. 1170-1181, 2011.

[77] V. S. Ramgolam, Y. Sha, J. Jin, X. Zhang, and S. Markovic-Plese, "IFN- $\beta$ inhibits human Th17 cell differentiation," The Journal of Immunology, vol. 183, no. 8, pp. 5418-5427, 2009.

[78] R. C. Axtell, B. A. de Jong, K. Boniface et al., "T helper type 1 and 17 cells determine efficacy of interferon- $\beta$ in multiple sclerosis and experimental encephalomyelitis," Nature Medicine, vol. 16, no. 4, pp. 406-412, 2010.

[79] R. C. Axtell, C. Raman, and L. Steinman, "Type i interferons: beneficial in Th1 and detrimental in Th17 autoimmunity," Clinical Reviews in Allergy and Immunology, vol. 44, no. 2, pp. 114-120, 2013.

[80] S. E. Bushnell, Z. Zhao, C. C. Stebbins et al., "Serum IL-17F does not predict poor response to IM IFN $\beta$-1a in relapsing-remitting MS," Neurology, vol. 79, no. 6, pp. 531-537, 2012.

[81] M. Tanaka, K. Tanaka, and M. Komori, "Interferon-beta(1b) treatment in neuromyelitis optica," European Neurology, vol. 62, no. 3, pp. 167-170, 2009.

[82] J. Shimizu, Y. Hatanaka, M. Hasegawa et al., "IFN $\beta$-1b may severely exacerbate Japanese optic-spinal MS in neuromyelitis optica spectrum," Neurology, vol. 75, no. 16, pp. 1423-1427, 2010.

[83] S.-H. Kim, W. Kim, X. F. Li, I.-J. Jung, and H. J. Kim, “Does interferon beta treatment exacerbate neuromyelitis optica spectrum disorder?” Multiple Sclerosis, vol. 18, no. 10, pp. 1480-1483, 2012.

[84] R. Aharoni, R. Eilam, A. Stock et al., "Glatiramer acetate reduces Th-17 inflammation and induces regulatory T-cells in the CNS of mice with relapsing-remitting or chronic EAE," Journal of Neuroimmunology, vol. 225, no. 1-2, pp. 100-111, 2010.

[85] R. Bergamaschi, C. Uggetti, S. Tonietti, M. G. Egitto, and V. Cosi, "A case of relapsing neuromyelitis optica treated with glatiramer acetate," Journal of Neurology, vol. 250, no. 3, pp. 359-361, 2003.

[86] K. Gartzen, V. Limmroth, and N. Putzki, "Relapsing neuromyelitis optica responsive to glatiramer acetate treatment," European Journal of Neurology, vol. 14, no. 6, pp. e12-e13, 2007.

[87] K.-C. Wang, C.-L. Lee, S.-Y. Chen, K.-H. Lin, and C.-P. Tsai, "Glatiramer acetate could be a hypothetical therapeutic agent for neuromyelitis optica," Medical Hypotheses, vol. 76, no. 6, pp. 820-822, 2011.

[88] M. Mehling, R. Lindberg, F. Raulf et al., "Th17 central memory T cells are reduced by FTY720 in patients with multiple sclerosis," Neurology, vol. 75, no. 5, pp. 403-410, 2010.

[89] D. K. Sato, I. Nakashima, A. Bar-Or et al., "Changes in Th17 and regulatory $\mathrm{T}$ cells after fingolimod initiation to treat multiple sclerosis," Journal of Neuroimmunology, vol. 268, no. 1-2, pp. 9598, 2014.

[90] J.-H. Min, B. J. Kim, and K. H. Lee, "Development of extensive brain lesions following fingolimod (FTY720) treatment in a patient with neuromyelitis optica spectrum disorder," Multiple Sclerosis, vol. 18, no. 1, pp. 113-115, 2012.
[91] S. Izaki, S. Narukawa, A. Kubota, T. Mitsui, H. Fukaura, and K. Nomura, "A case of neuromyelitis optica spectrum disorder developing a fulminant course with multiple white-matter lesions following fingolimod treatment," Clinical Neurology, vol. 53, no. 7, pp. 513-517, 2013.

[92] H. Zhang and A. S. Verkman, "Eosinophil pathogenicity mechanisms and therapeutics in neuromyelitis optica," The Journal of Clinical Investigation, vol. 123, no. 5, pp. 2306-2316, 2013.

[93] K. Sugita, K. Kabashima, J.-I. Sakabe, R. Yoshiki, H. Tanizaki, and Y. Tokura, "FTY720 regulates bone marrow egress of eosinophils and modulates late-phase skin reaction in mice," American Journal of Pathology, vol. 177, no. 4, pp. 1881-1887, 2010.

[94] C. H. Polman, P. W. O'Connor, and E. Havrdova, "A randomized, placebo-controlled trial of natalizumab for relapsing multiple sclerosis," The New England Journal of Medicine, vol. 354, pp. 899-910, 2006.

[95] I. Kleiter, K. Hellwig, A. Berthele et al., "Failure of natalizumab to prevent relapses in neuromyelitis optica," Archives of Neurology, vol. 69, no. 2, pp. 239-245, 2012.

[96] M. H. Barnett, J. W. Prineas, M. E. Buckland, J. Parratt, and J. D. Pollard, "Massive astrocyte destruction in neuromyelitis optica despite natalizumab therapy," Multiple Sclerosis, vol. 18, no. 1, pp. 108-112, 2012.

[97] J. Kitley, N. Evangelou, W. Küker, A. Jacob, M. I. Leite, and J. Palace, "Catastrophic brain relapse in seronegative NMO after a single dose of natalizumab," Journal of the Neurological Sciences, vol. 339, no. 1-2, pp. 223-225, 2014.

[98] H. Peng, M. Guerau-de-Arellano, V. B. Mehta et al., "Dimethyl fumarate inhibits dendritic cell maturation via nuclear factor $\kappa \mathrm{B}(\mathrm{NF}-\kappa \mathrm{B})$ and extracellular signal-regulated kinase 1 and $2(\mathrm{ERK} 1 / 2)$ and mitogen stress-activated kinase 1 (MSK1) signaling," The Journal of Biological Chemistry, vol. 287, no. 33, pp. 28017-28026, 2012.

[99] P. J. Darlington, T. Touil, J.-S. Doucet et al., "Diminished Th17 (not Th1) responses underlie multiple sclerosis disease abrogation after hematopoietic stem cell transplantation," Annals of Neurology, vol. 73, no. 3, pp. 341-354, 2013.

[100] F. Peng, W. Qiu, J. Li et al., "A preliminary result of treatment of neuromyelitis optica with autologous peripheral hematopoietic stem cell transplantation," The Neurologist, vol. 16, no. 6, pp. 375-378, 2010.

[101] P. Aouad, J. Li, C. Arthur, R. Burt, S. Fernando, and J. Parratt, "Resolution of aquaporin-4 antibodies in a woman with neuromyelitis optica treated with human autologous stem cell transplant," Journal of Clinical Neuroscience, vol. 22, no. 7, pp. 1215-1217, 2015.

[102] R. Greco, A. Bondanza, M. C. Oliveira et al., "Autologous hematopoietic stem cell transplantation in neuromyelitis optica: a registry study of the EBMT Autoimmune Diseases Working Party," Multiple Sclerosis, vol. 21, no. 2, pp. 189-197, 2015.

[103] M. Matiello, S. J. Pittock, L. Porrata, and B. G. Weinshenker, "Failure of autologous hematopoietic stem cell transplantation to prevent relapse of neuromyelitis optica," Archives of Neurology, vol. 68, no. 7, pp. 953-955, 2011.

[104] M. C. Papadopoulos, J. L. Bennett, and A. S. Verkman, “Treatment of neuromyelitis optica: state-of-the-art and emerging therapies," Nature Reviews Neurology, vol. 10, no. 9, pp. 493-506, 2014.

[105] C. de Andrés, R. Teijeiro, A. Saiz, P. Fernández, and S. SánchezRamón, "Changes in B and T-cell subsets and NMO-IgG levels 
after immunoglobulins and rituximab treatment for an acute attack of neuromyelitis optica," Neurologia, vol. 30, pp. 276-282, 2015.

[106] F. L. Van De Veerdonk, B. Lauwerys, R. J. Marijnissen et al., "The anti-CD20 antibody rituximab reduces the Th17 cell response," Arthritis and Rheumatism, vol. 63, no. 6, pp. 1507-1516, 2011.

[107] F. Ciccia, G. Guggino, A. Rizzo et al., "Rituximab modulates IL-17 expression in the salivary glands of patients with primary Sjögren's syndrome," Rheumatology, vol. 53, no. 7, pp. 1313-1320, 2014.

[108] B. Szalay, B. Vásárhelyi, Á. Cseh et al., "The impact of conventional DMARD and biological therapies on CD4+ cell subsets in rheumatoid arthritis: a follow-up study," Clinical Rheumatology, vol. 33, no. 2, pp. 175-185, 2014.

[109] Y. Li, L. Jiang, S. Zhang et al., "Methotrexate attenuates the Th17/IL-17 levels in peripheral blood mononuclear cells from healthy individuals and RA patients," Rheumatology International, vol. 32, no. 8, pp. 2415-2422, 2012.

[110] G. Guggino, A. Giardina, A. Ferrante et al., "The in vitro addition of methotrexate and/or methylprednisolone determines peripheral reduction in Th17 and expansion of conventional Treg and of IL-10 producing Th17 lymphocytes in patients with early rheumatoid arthritis," Rheumatology International, vol. 35, no. 1, pp. 171-175, 2015.

[111] B. M. Segal, C. S. Constantinescu, A. Raychaudhuri, L. Kim, R. Fidelus-Gort, and L. H. Kasper, "Repeated subcutaneous injections of IL12/23 p40 neutralising antibody, ustekinumab, in patients with relapsing-remitting multiple sclerosis: a phase II, double-blind, placebo-controlled, randomised, dose-ranging study," The Lancet Neurology, vol. 7, no. 9, pp. 796-804, 2008.

[112] G. Elain, K. Jeanneau, A. Rutkowska, A. K. Mir, and K. K. Dev, "The selective anti-IL17A monoclonal antibody secukinumab (AIN457) attenuates IL17A-induced levels of IL6 in human astrocytes," Glia, vol. 62, no. 5, pp. 725-735, 2014.

[113] D. D. Patel, D. M. Lee, F. Kolbinger, and C. Antoni, "Effect of IL-17A blockade with secukinumab in autoimmune diseases," Annals of the Rheumatic Diseases, vol. 72, supplement 2, pp. iill6-iil23, 2013.

[114] E. Havrdová, A. Belova, and A. Goloborodko, "Positive proof of concept of AIN457, an antibody against interleukin-17A, in relapsing-remitting multiple sclerosis," Multiple Sclerosis Journal, vol. 18, no. 4, pp. 509-520, 2012.

[115] A. Deiß, I. Brecht, A. Haarmann, and M. Buttmann, "Treating multiple sclerosis with monoclonal antibodies: a 2013 update," Expert Review of Neurotherapeutics, vol. 13, no. 3, pp. 313-335, 2013.

[116] M. Montes, X. Zhang, L. Berthelot et al., "Oligoclonal myelinreactive T-cell infiltrates derived from multiple sclerosis lesions are enriched in Th17 cells," Clinical Immunology, vol. 130, no. 2 , pp. 133-144, 2009. 


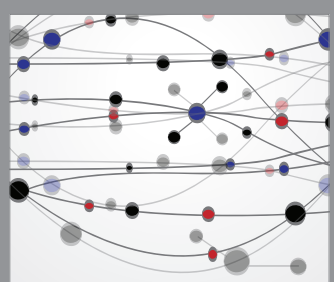

The Scientific World Journal
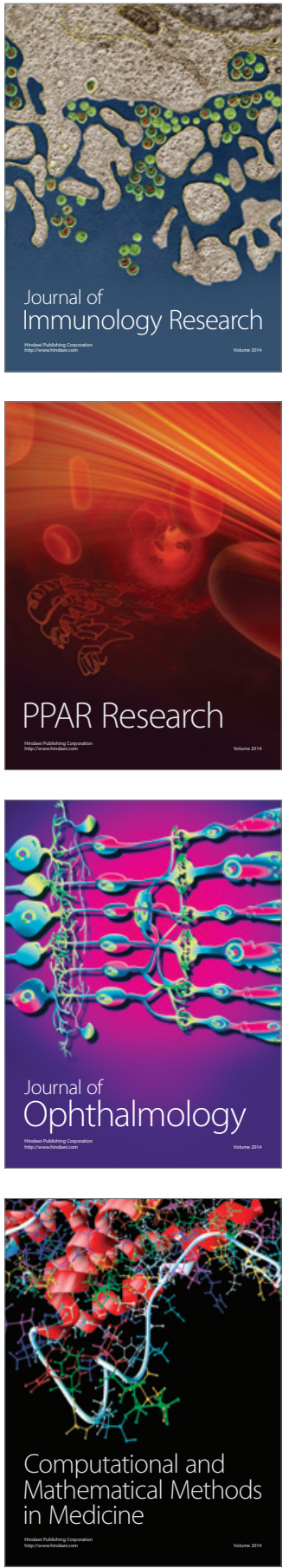

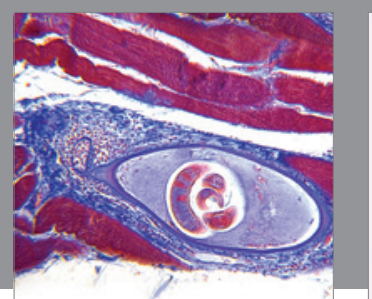

Gastroenterology Research and Practice

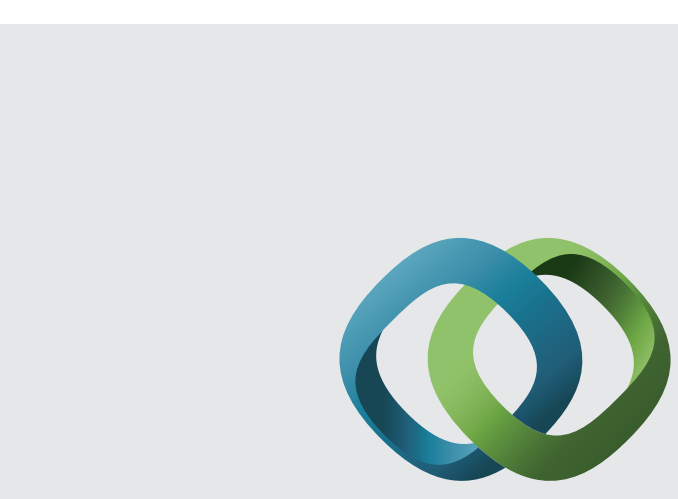

\section{Hindawi}

Submit your manuscripts at

http://www.hindawi.com
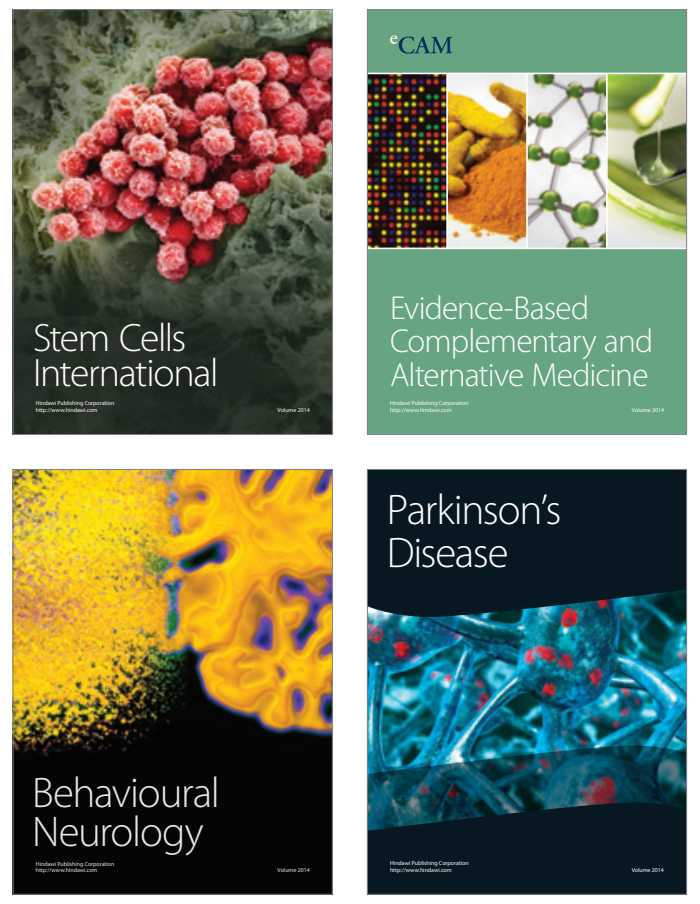
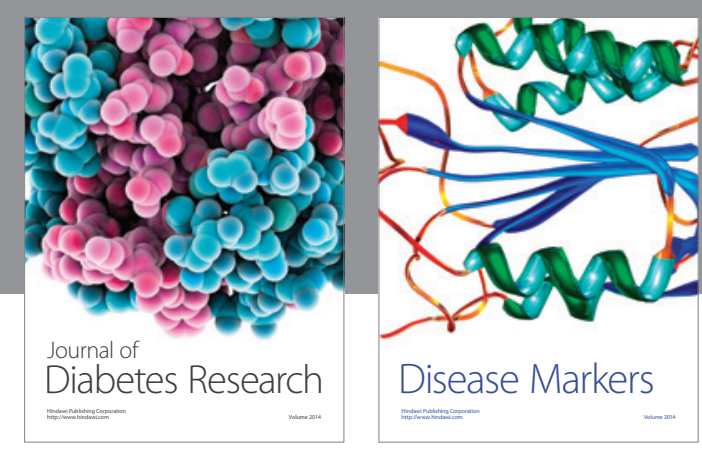

Disease Markers
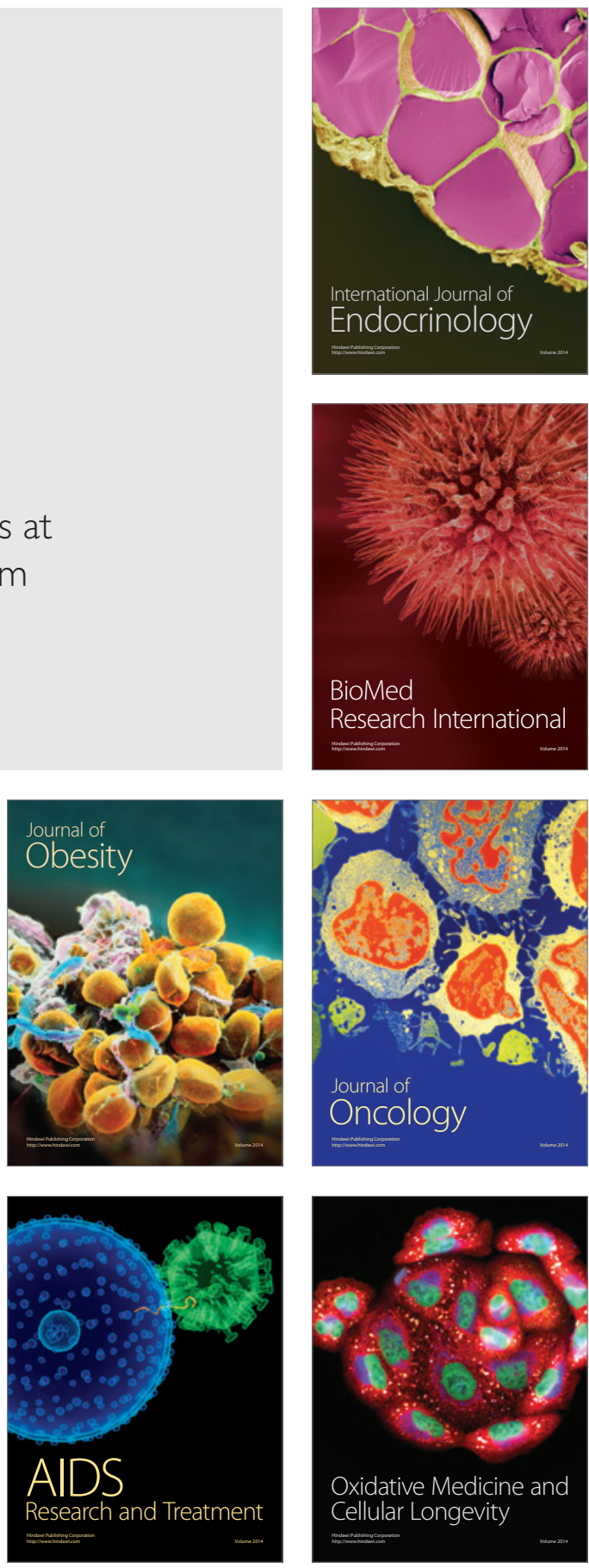